\section{VALIDACIÓN DE LA VERSIÓN PERUANA DEL PHQ-9 PARA EL DIAGNÓSTICO DE DEPRESIÓN}

\section{VALIDATION OF THE PERUVIAN VERSION OF THE PHQ-9 FOR DIAGNOSING DEPRESSION}

\author{
María Calderón ${ }^{1, a}$, Juan Antonio Gálvez- \\ Buccollini2,3,b, Gloria Cueva ${ }^{4, b}$, Carlos Ordoñez ${ }^{5, b}$, \\ Carlos Bromley ${ }^{4, b}$, Fabián Fiestas ${ }^{1, c}$
}

Sr. Editor. Las enfermedades psiquiátricas explican el $16 \%$ de los años de vida saludable perdidos por discapacidad o muerte prematura en el Perú, lo que coloca a este grupo de enfermedades como la causa más importante de carga de enfermedad en el país. De estas, la depresión unipolar es una de las más importantes, junto con los problemas relacionados al consumo de alcohol ${ }^{(1)}$. El problema de las enfermedades mentales se ve agudizado debido al reducido número de psiquiatras, y su distribución muy desigual en las regiones, con una concentración mayor en Lima, ciudad capital.

Ha quedado establecido que el $90 \%$ de los problemas psiquiátricos pueden ser manejados en la atención primaria de salud (2), por ello la detección y manejo por parte de los médicos no psiquiatras constituye un gran apoyo para enfrentar la depresión unipolar en las poblaciones. Sin embargo, se ha evidenciado que los médicos no psiquiatras no reconocen los síntomas ni establecen el tratamiento adecuado ${ }^{(3)}$.

Para lograr que más personas puedan acceder a tratamientos de calidad para depresión, es importante contar con instrumentos diagnósticos validados localmente. El PHQ-9 (Patient Health Questionnarie-9) es una prueba de tamizaje de depresión que ha sido validado en muchos países, incluyendo Chile ${ }^{(4,5)}$. La principal ventaja del PHQ-9 sobre otros instrumentos de tamizaje para depresión es su rapidez; puede ser autoaplicado y, además de ser una herramienta diagnóstica, también indica la gravedad del cuadro, por lo que serviría para hacer un seguimiento del manejo y evolución de cada paciente.

\footnotetext{
Unidad de Análisis y Generación de Evidencias en Salud Pública, Instituto Nacional de Salud. Lima, Perú.

2 Harvard Medical School. Boston, EE. UU.

3 Veterans Affairs Boston Healthcare System. Boston, EE. UU.

4 Dirección de Salud Mental, Ministerio de Salud del Perú. Lima, Perú

5 Hospital Hermilio Valdizán. Lima, Perú

a Médico-cirujano, ${ }^{\mathrm{b}}$ médico psiquiatra, ${ }^{\mathrm{c}}$ médico epidemiólogo Recibido: 30-08-12 Aprobado: 17-10-12
}

Citar como: Calderón M, Gálvez-Buccollini JA, Cueva G, Ordoñez C, Bromley C, Fiestas F. Validación de la versión peruana del PHQ-9 para el diagnóstico de depresión. Rev Peru Med Exp Salud Publica. 2012;29(4):578-9.
Aunque los creadores del instrumento presentaron una versión oficial para el Perú (disponible en Patient Health Questionnaire (PHQ) Screeners), este instrumento aún no ha sido validado; es por ello que la Dirección de Salud Mental del Ministerio de Salud del Perú (MINSA), en coordinación con el Instituto Nacional de Salud (INS), dentro del marco del desarrollo de "Algoritmos de Diagnóstico y Tratamiento de Depresión" ha realizado la validación por juicio de expertos del PHQ-9, como primer paso para su validación en el contexto peruano. Esta validación se realizó en una reunión técnica con la presencia de especialistas en salud mental, incluyendo 23 psiquiatras, 3 psicólogos y una enfermera.

En general, la percepción entre los especialistas fue que el PHQ-9 es un buen instrumento para el diagnóstico de depresión, y que puede ser de fácil uso en el contexto del sistema de atención primaria en el Perú. Algunos comentarios en el sentido de perfeccionar el instrumento incluyeron los siguientes: i) especificar el número de días para la clasificación de "para nada", "varios días", "más de la mitad de los días" y "casi todo los días"; con el fin de que sea más entendible para la mayoría de pacientes; ii) en el ítem 2, añadir la palabra "triste" para reforzar el concepto de "deprimido"; iii) en el ítem 7, se recomendó no especificar la palabra "periódico", y dejar el ítem fraseado de la siguiente manera: "Dificultad para poner atención o concentrarse en las cosas, tales como leer o ver televisión"; iv) en el ítem 9 debe añadirse la frase "hacerse daño" ya que la palabra "lastimarse" no es de amplio uso en el contexto peruano.

En conclusión, el PHQ-9 es un instrumento válido para diagnosticar depresión, a juicio de los expertos, para ser aplicado en el Perú. En el futuro sigue una fase de validación de criterio en poblaciones costeñas, de la sierra y de la selva, que además permita detectar variaciones en la sensibilidad y especificidad del instrumento dependientes de los contextos socioculturales que existen en el país.

Fuentes de financiamiento: autofinanciado.

Conflictos de interés: los autores declaran no tener conflictos de interés.

\section{REFERENCIAS BIBLIOGRÁFICAS}

1. Velásquez A. La carga de enfermedad y lesiones en el Perú y las prioridades del plan esencial de aseguramiento universal. Rev Peru Med Exp Salud Publica. 2009;26(2):222-31.

2. Organización Panamericana de la Salud. Marco de referencia para la implementación de la Estrategia Regional de Salud Mental. Washington, DC: OPS; 2011. 
3. Galvez-Buccollini JA, Fiestas F. Necesidad de evaluar las guías clínicas peruanas de tratamiento para trastornos mentales [carta]. Rev Peru Med Exp Salud Publica. 201 1; 28(4):698-9.

4. Gilbody S, Richards D, Brealey S, Hewitt C. Screening for depression in medical setting with the Patient Health Questionnaire (PHQ): a diagnostic meta-analysis. J Gen Intern Med. 2007;22(11):1596-602.

5. Baader T, Molina JL, Venezian S, Rojas C, Farías R, FierroFreixenet $\mathrm{C}$, et al. Validación y utilidad de la encuesta PHQ-9 (Patient Health Questionnaire) en el diagnostic de depression en pacientes usuarios de atención primaria en Chile. Rev Chil Neuro-Psiquiat. 2012;50(1):10-22.

Correspondencia: Fabian Fiestas

Dirección: Capac Yupanqui 1400, Lima 11, Perú.

Teléfono: (+511) 985-765-743

Correoelectrónico:ffiestas@epi.msu.edu

\section{NECESIDAD DE VIGILAR LA RESISTENCIA DEL $P$. falciparum AL ARTESUNATO EN EL PERÚ}

\author{
A NEED TO MONITOR P. falciparum \\ RESISTANCE TO ARTESUNATE IN PERU
}

\section{Salomón Durand ${ }^{1,2, a}$, Moisés Sihuinchaa,a, Arnaldo Lachira $^{3, a}$, Jorge Chaves ${ }^{3, a}$, César Cabezas ${ }^{4, a}$}

Sr. Editor. A finales del 2001, el Perú se convirtió en el primer país de las Américas en adoptar la terapia combinada (TC) con mefloquina/artesunato (MQ/AS) y sulfadoxina pirimetamina/artesunato (SP/AS) como terapia de primera línea para la malaria no complicada por $P$. falciparum. Posteriormente, Bolivia, Brasil, Guyana y Ecuador cambian también su esquema de tratamiento a la TC.

Ante la amenaza de la propagación de cepas resistentes a partir del sudeste asiático, en donde últimamente se ha demostrado retardo en el aclaramiento de la parasitemia y resistencia in vitro e in vivo a la artemisinina ${ }^{(1)}$, realizamos una revisión a partir de la

\footnotetext{
1 Centro de Investigación de Enfermedades Tropicales de la Marina de los EE. UU. (NAMRU-6). Iquitos, Perú.

2 Hospital de Iquitos "César Garayar García", Dirección Regional de Salud Loreto. Iquitos, Perú.

3 Dirección Regional de Salud Piura. Piura, Perú.

4 Instituto Nacional de Salud. Lima, Perú.

a Médico infectólogo-tropicalista

Recibido: 05-10-12 Aprobado: 31-10-12
}

Citar como: Durand S, Sihuincha M, Lachira A, Chaves J, Cabezas C. Necesidad de vigilar la resistencia del P. Falciparum al artesunato en el Perú. Rev Peru Med Exp Salud Publica. 2012;29(4):579-80. base datos MEDLINE, en busca de estudios realizados en nuestro país. Se buscó conocer la rapidez de acción del artesunato sobre la carga parasitaria, establecer un registro basal que sirva como punto de comparación para estudios futuros de eficacia, e identificar los factores clave donde se podría intervenir para evitar la propagación de la resistencia, en caso que esta cepa se introdujera en el Perú o para evitar que se genere en nuestra región.

Se encontraron cinco estudios sobre la eficacia de la TC en el Perú publicados entre el 2000 y 2006. El primer problema para compararlos fueron los días de seguimiento, ya que hasta el 2004, usualmente, se realizaba seguimiento por 28 días, pero en vista del prolongado tiempo de vida media de la mefloquina, posteriormente, se recomendó seguir al paciente por más de 28 días. Hasta el 2006, fecha del último estudio revisado, la TC fue muy eficaz (98-100\%), aun en el seguimiento a 63 días, y la tasa de parasitemia persistente al día 3 fue menor al 3\%. (Tabla 1).

En el estudio de Macedo et al. (2) se evaluó el esquema actual utilizado en el Perú, el cual consiste en administrar AS el primer día y MQ/AS el segundo y tercer día. Se evidenció así el efecto a las 24 horas del AS usado como monoterapia. En los otros estudios, los pacientes recibieron el esquema MQ/AS el primer y segundo día y AS el tercer día. A las 24 horas, el porcentaje de láminas positivas fue $31 \%$, y a las 48 horas (ya agregada la $M Q$ ) fue de $5 \%$. Lo hallado difiere de lo descrito por Rogers

Tabla 1. Resultados de los estudios de eficacia de TC para el tratamiento de la malaria por $P$. falciparum no complicada realizados en el Perú, 2000-2006.

\begin{tabular}{|c|c|c|c|c|c|c|c|}
\hline & \multirow[b]{2}{*}{ esquema } & \multicolumn{6}{|c|}{$\begin{array}{l}\text { Porcentaje de láminas positivas } \\
\text { durante el seguimiento }\end{array}$} \\
\hline & & $\begin{array}{c}\text { día } \\
1 \\
\end{array}$ & $\begin{array}{c}\text { día } \\
2\end{array}$ & $\begin{array}{c}\text { día } \\
3\end{array}$ & $\begin{array}{l}\text { día } \\
28 \\
\end{array}$ & $\begin{array}{l}\text { día } \\
56\end{array}$ & $\begin{array}{l}\text { día } \\
63 \\
\end{array}$ \\
\hline $\begin{array}{l}\text { Macedo et } \\
\text { al. }(2006)^{(2)}\end{array}$ & MQ/AS & $\begin{array}{l}31 / 100 \\
(31,0 \%)\end{array}$ & $\begin{array}{l}5 / 100 \\
(5,0 \%)\end{array}$ & $\begin{array}{l}1 / 100 \\
(1,0 \%)\end{array}$ & $\begin{array}{c}1 / 96 \\
(0,9 \%)^{*}\end{array}$ & - & - \\
\hline $\begin{array}{l}\text { Gutman et } \\
\text { al. }(2004)^{(3)}\end{array}$ & MQ/AS & $\begin{array}{c}3 / 38 \\
(7,8 \%)\end{array}$ & $\begin{array}{c}2 / 38 \\
(5,2 \%)\end{array}$ & $\begin{array}{c}1 / 38 \\
(2,6 \%)\end{array}$ & $0 / 38$ & $0 / 38$ & - \\
\hline \multirow[t]{2}{*}{$\begin{array}{l}\text { Grande et } \\
\text { al. }(2005)^{(4)}\end{array}$} & MQ/AS & $\begin{array}{l}108 / 260 \\
(42,5 \%)\end{array}$ & $\begin{array}{l}10 / 260 \\
(3,8 \%)\end{array}$ & 0 & - & - & $\begin{array}{l}1 / 236 \\
(0,4 \%)\end{array}$ \\
\hline & DHA/PPQ & $\begin{array}{l}84 / 260 \\
(32,3 \%)\end{array}$ & $\begin{array}{l}3 / 260 \\
(1,9 \%)\end{array}$ & 0 & - & - & $\begin{array}{l}4 / 230 \\
(1,7 \%)\end{array}$ \\
\hline $\begin{array}{l}\text { Marquino et } \\
\text { al. }(2000)^{(5)}\end{array}$ & SPIAS & - & - & $\begin{array}{c}2 / 94 \\
(2,1 \%)\end{array}$ & $\begin{array}{c}1 / 94 \\
(1,0 \%)\end{array}$ & - & - \\
\hline \multirow[t]{2}{*}{$\begin{array}{l}\text { Marquino et } \\
\text { al. }(2000)^{(6)}\end{array}$} & MQ/AS & - & $\begin{array}{c}9 / 51 \\
(17,6 \%)\end{array}$ & $\begin{array}{c}1 / 51 \\
(1,9 \%)\end{array}$ & $0 / 51$ & - & - \\
\hline & $M Q$ & - & $\begin{array}{c}34 / 47 \\
(72,3 \%)\end{array}$ & $\begin{array}{c}12 / 47 \\
(25,5 \%)\end{array}$ & $0 / 47$ & - & - \\
\hline
\end{tabular}

* En el examen de genotipificación se halló que se trataba de una cepa diferente, probable reinfección.

$M Q / A S:$ mefloquina/artesunato; SP/AS: sulfadoxina pirimetamina/artesunato; DHA/PPQ: dihidroartemisinina/piperaquina. 